\title{
Inhibitory actions of cilostazol on electrical responses of smooth muscle isolated from the guinea-pig stomach antrum
}

\author{
Eri NAKAMURA ${ }^{1}$, Ayako HASHIMOTO ${ }^{2}$, Yoshihiko KITO', \\ Hikaru HASHITANI ${ }^{1}$, Toyoki MORI ${ }^{2}$ and Hikaru SUZUKI ${ }^{1}$ \\ ${ }^{1}$ Department of Physiology, Nagoya City University Medical School, Mizuho-ku, Nagoya \\ 467-8601, Japan \\ ${ }^{2}$ Research Institute of Pharmacological \& Therapeutical Development, Otsuka \\ Pharmaceutical Co., Ltd., Kawauchi-cho, Tokushima 771-0192, Japan
}

\begin{abstract}
We have investigated the effects of cilostazol, a type III phosphodiesterase inhibitor, on the electrical responses of smooth muscle tissue isolated from the guinea-pig stomach antrum. Cilostazol $\left(10^{-5} \mathrm{M}\right)$ inhibited slow waves recorded from circular muscle cells, but did not significantly alter the pacemaker potentials and follower potentials recorded from myenteric interstitial cells and longitudinal muscle cells respectively. Slow potentials generated in isolated circular muscle bundles without attached myenteric interstitial cells were inhibited by cilostazol $\left(>10^{-7} \mathrm{M}\right)$, while all membrane activities were abolished by $10^{-5} \mathrm{M}$ cilostazol. In circular muscle bundles, the input resistance of smooth muscle cells and the refractory period for the generation of slow potentials were not altered during the inhibition of spontaneous activity with cilostazol. While cilostazol at $10^{-7}$ and $10^{-6} \mathrm{M}$ did not elevate the tissue content of cyclic AMP, at $10^{-5} \mathrm{M}$ cyclic AMP was elevated by about $30 \%$. A similar elevation was also produced by $10^{-7} \mathrm{M}$ forskolin. The content of cyclic AMP was not significantly increased in preparations stimulated with $10^{-3} \mathrm{M}$ caffeine. The potency for inhibiting slow waves was in the order caffeine $\left(10^{-3} \mathrm{M}\right)$ $>$ forskolin $\left(10^{-7} \mathrm{M}\right)>$ cilostazol $\left(10^{-5} \mathrm{M}\right)$. The frequency of slow waves was decreased by caffeine or forskolin but not by cilostazol, while the duration was reduced by caffeine but not by cilostazol or forskolin. Follower potentials were modulated by caffeine and forskolin, but not by cilostazol: the duration was reduced by caffeine, the frequency was reduced by caffeine or forskolin, and the amplitude was not significantly altered by any of them. The results indicate that cilostazol has high selectivity in inhibiting the activity of circular muscle much more than that of longitudinal muscle or pacemaker cells, with no causal relation to the tissue content of cyclic AMP as appears to be the case for the inhibitory actions of caffeine and forskolin.
\end{abstract}

Key words: cilostazol, caffeine, forskolin, gastric muscle, cyclic AMP

Correspondence to: Hikaru Suzuki, Department of Physiology, Nagoya City University Medical School Mizuho-ku, Nagoya 467-8601, Japan

Phone: +81-52-853-8129 Fax: +81-52-842-1538 e-mail: hisuzuki@med.nagoya-cu.ac.jp 


\section{Introduction}

Gastric smooth muscle is spontaneously active with rhythmic generation of either slow waves or action potentials or both, but with only the action potentials and not the slow waves inhibited by chemicals which block voltage-gated L-type Ca-channels (Tomita, 1981). Although the ionic basis of the generation of slow waves remains controversial, the potentials may originate in interstitial cells of Cajal that are distributed in the myenteric region (ICC-MY) (Sanders, 1996; Huizinga et al., 1997; Sanders et al., 1999; Suzuki, 2000; Hirst and Ward, 2003; Takaki, 2003). ICC-MY distributed within the muscle tissue form gap junctions with surrounding ICC-MY and smooth muscle cells (Komuro et al., 1996), and pacemaker potentials produced in the ICC-MY are propagated to smooth muscle cells in an electrotonic manner (Dickens et al., 1999).

In isolated smooth muscle tissue of the guinea-pig gastric antrum, three types of electrical responses are recorded; slow waves from circular smooth muscle cells, driving potentials (or pacemaker potentials) from ICC-MY and square-shaped potentials (follower potentials) from longitudinal smooth muscle cells (Dickens et al., 1999). Each of these potentials is synchronized, with the pacemaker potentials appearing prior to slow waves or follower potentials, indicating that ICC-MY are indeed pacing the rhythmic activities of circular and longitudinal smooth muscle cells (Dickens et al., 1999). However, isolated circular muscle bundles, in the absence of ICC-MY, also produce regenerative potentials (slow potentials) rhythmically (Suzuki and Hirst, 1999). These two types of potentials, slow potentials and slow waves, are generated under different conditions of the circular muscle, and although both are voltage-sensitive, as depolarization accelerates and hyperpolarization inhibits their generation (Nose et al., 2000; Fukuta et al., 2002), the frequency is much higher for slow waves (3-6 cycle per min, cpm) than for slow potentials (0.1-2 cpm) (Suzuki and Hirst, 1999; Nose et al., 2000). Significant difference between these two types of potentials appears in their sensitivity to caffeine, as low concentrations of caffeine $(1 \mathrm{mM})$ abolish slow potentials but only reduce the amplitude of slow waves by about half (Dickens et al., 1999; Suzuki and Hirst, 1999; Nose et al., 2000). Caffeine has inhibitory actions on smooth muscle cells, but the cellular mechanism of the inhibition is controversial. In general, the inhibitory actions of caffeine are considered to be produced by the elevation of the tissue concentration of cyclic AMP (cAMP), as a result of the inhibition of phosphodiesterase activity (Arnoud, 1987). However, caffeine has been reported to have possible inhibitory actions on inositol 1,4,5-trisphosphate $\left(\mathrm{IP}_{3}\right)$ receptors in a variety of tissues (Parker and Ivorra, 1991; Toescu et al., 1992; Berridge, 1993; Somlyo and Somlyo, 1994; Maes et al., 1999).

Cilostazol is a known inhibitor of type III phosphodiesterase with antithrombotic and vasodilatory actions, due to an elevated tissue level of cAMP (Kawamura et al., 1985; Kimura et al., 1985; Kohda et al., 1999), and its clinical importance is apparent in the treatment of arterial circulation disorders (Kobayashi et al., 1985; Yasuda et al., 1985; Tanaka et al., 1989; Dawson et $a l .$, 1998). Increased cAMP levels may have multiple effects on smooth muscle cells, such as an enhanced activity of Ca-channels, accelerated reduction of $\left[\mathrm{Ca}^{2+}\right]_{i}$ due to activation of the Capump at the internal membrane and enhanced phosphorylation of some proteins through 
activation of A-kinase (Abdel-Latif, 1996; Kuriyama et al., 1998; Tertyshnikova and Fein, 1998). In intestinal smooth muscle, an increase in cAMP levels either directly or indirectly inhibits spontaneous activity (Huizinga et al., 1991; Koh et al., 2000; Kito and Suzuki, 2003c). This seems to be also the case for gastric smooth muscle, and chemicals known to inhibit phosphodiesterases such as caffeine and forskolin have inhibitory actions in the guinea-pig stomach (Ozaki et al., 1992; Tsugeno et al., 1995; Kito et al., 2002a; Kito and Suzuki, 2003a). Therefore, it was considered that the actions of cilostazol on gastric smooth muscle cells may, if any, help explain the actions of caffeine.

Experiments were carried out to investigate the possible involvement of cAMP during cilostazol stimulation of smooth muscle isolated from the guinea-pig stomach antrum. A comparison was made of the effects of cilostazol on electrical activity with the level of cAMP in gastric smooth muscle. The results indicated that cilostazol selectively inhibits the activity of circular muscle cells, with no significant causal relation to the tissue content of cAMP.

\section{Methods}

\section{Preparations}

Male albino guinea-pigs, weighing 200-300 g, were anesthetized with fluoromethyl 2,2,2trifluoro-1-(trifluoromethyl) ethyl ether (sevoflurane, Maruishi Pharm., Osaka, Japan), and exsanguinated by decapitation. All animals were treated ethically according to the Guidelines for the Care and Use of Laboratory Animals of the Nagoya City University Medical School, as accredited by The Physiological Society of Japan. The stomach was excised, and opened in Krebs solution by cutting along the small curvature. The mucosal layers were removed by cutting with fine scissors, and smooth muscle tissue was removed from the antrum region.

A segment of smooth muscle tissue (about $1.5 \mathrm{~mm}$ width and $3 \mathrm{~mm}$ long) was isolated and three types of preparation prepared. The intact tissue preparation had both circular and longitudinal muscle layers $(500 \mu \mathrm{m} \times 500 \mu \mathrm{m})$, and was pinned out with the mucosal side uppermost on a silicone rubber plate fixed at the bottom of the organ bath $(8 \mathrm{~mm}$ wide, $8 \mathrm{~mm}$ deep, $20 \mathrm{~mm}$ long). The longitudinal muscle preparation (300 $\mu \mathrm{m} \times 500 \mu \mathrm{m})$ was prepared by removing the circular muscle, and the segment pinned out with the mucosal side uppermost on the silicone rubber plate. The isolated circular muscle bundle preparation (250-300 $\mu \mathrm{m}$ long) was made by removing the longitudinal muscle layer with the attached myenteric plexus, and then pinning out with the serosal side uppermost on the silicone rubber plate. These tissues were superfused with warmed $\left(35^{\circ} \mathrm{C}\right)$ and oxygenated Krebs solution, at a constant flow rate of about $2 \mathrm{ml} / \mathrm{min}$.

Experiments were carried out in the presence of $1 \mu \mathrm{M}$ nifedipine throughout, so as to minimize muscle movements. Conventional microelectrode techniques were used to record electrical activities of smooth muscle cells, and usually they were recorded from two cells simultaneously using two glass capillary microelectrodes (outer diameter, $1.2 \mathrm{~mm}$, inner diameter $0.6 \mathrm{~mm}$; Hilgenberg, Germany) filled with $0.5 \mathrm{M} \mathrm{KCl}$ (the tip resistances ranging between 200 and $300 \mathrm{M} \Omega$ ). In some experiments, responses were recorded using single electrodes. Electrical responses recorded with a high input impedance amplifier (Axoclamp-2B, 
Axon Instruments, Inc., Foster City, California, U.S.A.) were displayed on a cathode-ray oscilloscope (SS-7602, Iwatsu, Osaka, Japan) and also stored on a personal computer for later analysis.

The ionic composition of the Krebs solution was as follows (mM): $\mathrm{Na}^{+} 137.4, \mathrm{~K}^{+} 5.9, \mathrm{Ca}^{2+} 2.5$, $\mathrm{Mg}^{2+} 1.2, \mathrm{HCO}_{3}{ }^{-} 15.5, \mathrm{H}_{2} \mathrm{PO}_{4}^{-} 1.2, \mathrm{Cl}^{-} 134$, and glucose 11.5 . The solutions were aerated with $\mathrm{O}_{2}$ containing $5 \% \mathrm{CO}_{2}$, and the $\mathrm{pH}$ of the solutions was maintained at 7.2-7.3.

Strips of antrum muscle were incubated with cilostazol and forskolin for $10 \mathrm{~min}$, or caffeine for $5 \mathrm{~min}$, in a warmed $\left(35^{\circ} \mathrm{C}\right)$ and oxygenated Krebs solution, and the cAMP content of the tissue measured using the cAMP Biotrak Enzymeimmunoassay (EIA) system (Amersham Biosciences). Briefly, the incubated tissues were homogenized in the assay buffer of the cAMPEIA kit (Amersham Biosciences) at $10 \mathrm{mg} / \mathrm{mL}$, and centrifuged at $2,000 \times \mathrm{g}$ for $15 \mathrm{~min}$ at $4^{\circ} \mathrm{C}$. The supernatant was collected and used for the assay of cAMP. One hundred microliter of lysate or cAMP standard was placed on a donkey anti-rabbit IgG coated EIA plate, and $100 \mu \mathrm{L}$ of rabbit anti-cAMP was added to wells. After incubation for 2 hours at $4^{\circ} \mathrm{C}, 50 \mu \mathrm{L}$ of cAMPperoxidase conjugate was added and incubated for 1 hour at $4^{\circ} \mathrm{C}$. After the reaction mixture was aspirated, the wells were washed four times, and $150 \mu \mathrm{L}$ of enzyme substrate (TMB) was dispensed. After incubation for 1 hour at room temperature, $100 \mu \mathrm{L}$ of $1.0 \mathrm{M}$ sulfuric acid was added to each well to stop the reaction. Absorbance at $450 \mathrm{~nm}$ was measured using a microplate reader (Molecularr Devices Corporation) and the concentration of cAMP calculated by comparison with standard solutions using SOFTmax ${ }^{\mathrm{TM}}$ for Windows (Molecular Devices Corporation). Tissues stimulated with Krebs solution alone and those stimulated with DMSO (0.1\%)-containing solution were prepared as controls, the former for caffeine and the latter for cilostazol and forskolin.

Drugs used were caffeine, nifedipine, forskolin (from Calbiochem, San Diego, California, U.S.A.) and 6-[4-(1-cyclohexyl-1H-tetrazol-5-yl) butoxy]-3,4-dihydro-2(1H) -quinolinone (cilostazol, Otsuka Pharm. Co. Ltd., Tokyo, Japan). Forskolin, nifedipine and cilostazol were dissolved in dimethyl sulphoxide (DMSO) to make stock solutions, and they were added to Krebs solution immediately prior to use to make the desired concentrations. Other drugs tested were dissolved in distilled water. The final concentration of the solvent in Krebs solution did not exceed 1/1,000. Addition of these chemicals to Krebs solution did not alter the $\mathrm{pH}$ of the solution.

Experimental values were expressed as the mean value \pm standard deviation (SD) or standard error of mean (SEM). Statistical significance was tested using either the Student's $t$ test or the Williams test, and probabilities of less than $5 \%(\mathrm{P}<0.05)$ were considered to be significant.

\section{Results}

\section{Effects of cilostazol on electrical activity of gastric smooth muscle tissue}

In intact tissue of the stomach antrum, experiments were carried out to observe the effects of $10^{-5} \mathrm{M}$ cilostazol on slow waves, follower potentials and pacemaker potentials recorded from circular muscle cells, longitudinal muscle cells and ICC-MY, respectively. Cilostazol inhibited 


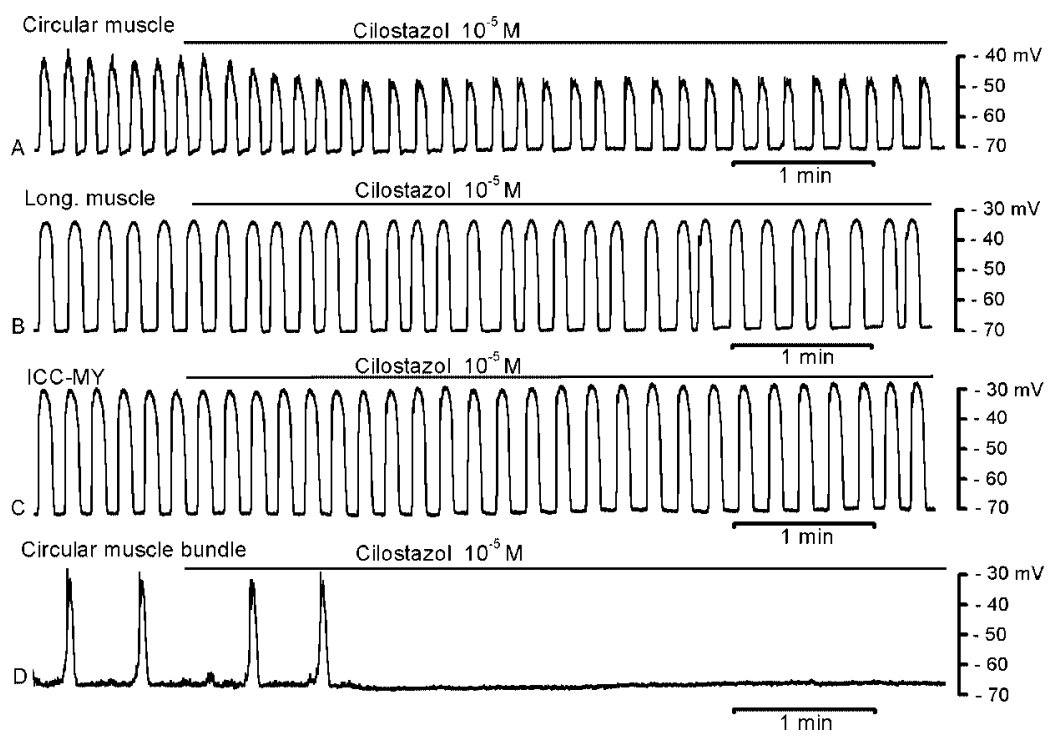

Fig. 1. Effects of cilostazol on spontaneous electrical activity of gastric smooth muscle cells. Spontaneous electrical responses were recorded from a circular muscle cell (A), a longitudinal muscle cell (B) and ICC-MY (C) in intact tissue and from an isolated circular muscle bundle (D). Cilostazol $\left(10^{-5} \mathrm{M}\right)$ was present for the duration of the bar shown above each record. A-D were recorded from different preparations.

slow waves, with no significant alteration to follower potentials and pacemaker potentials (Fig. 1). Slow waves are formed by two components: an electrotonic potential of pacemaker potentials, with superimposed slow potentials generated in the circular muscle (Hirst and Ward, 2003; Kito and Suzuki, 2003b). Therefore, it was considered that the inhibition of the slow waves by cilostazol may be largely the result of its action on the activity of the circular muscle. The effects of cilostazol were then examined on the slow potentials generated in isolated circular smooth muscle preparations. The slow potentials were observed to be abolished by cilostazol, with no significant alteration to the resting membrane potential (Fig. 1D). This inhibition by cilostazol of slow waves or slow potentials was reversible, but requiring up to 30 min to wash out (data not shown).

In isolated circular muscle preparations, the effects of increasing concentrations of cilostazol on slow potentials were observed, and the results summarized in Fig. 2. The inhibitory actions of cilostazol were evident on the amplitude (Fig. 2F), duration (Fig. 2G) and frequency (Fig. 2H) of the slow potentials at concentrations of higher than $10^{-7} \mathrm{M}$. In 8 preparations tested, slow potentials were abolished at $10^{-6} \mathrm{M}$ in 4 preparations and at $10^{-5} \mathrm{M}$ in 4 preparations.

The effects of cilostazol on slow potentials evoked by depolarizing pulses were observed, to test if cilostazol altered the refractory period for the generation of slow potentials (Kito et al., $2002 \mathrm{~b}$ ). In isolated small segments of circular smooth muscle, simultaneously recorded electrical responses from two different cells were synchronized, indicating that both cells were electrically coupled. One of the typical results is shown in Fig. 3, where $10^{-7} \mathrm{M}$ cilostazol reduced the frequency of spontaneously generated slow potentials (control, $4.47 \pm 0.50 \mathrm{cpm}$; in 

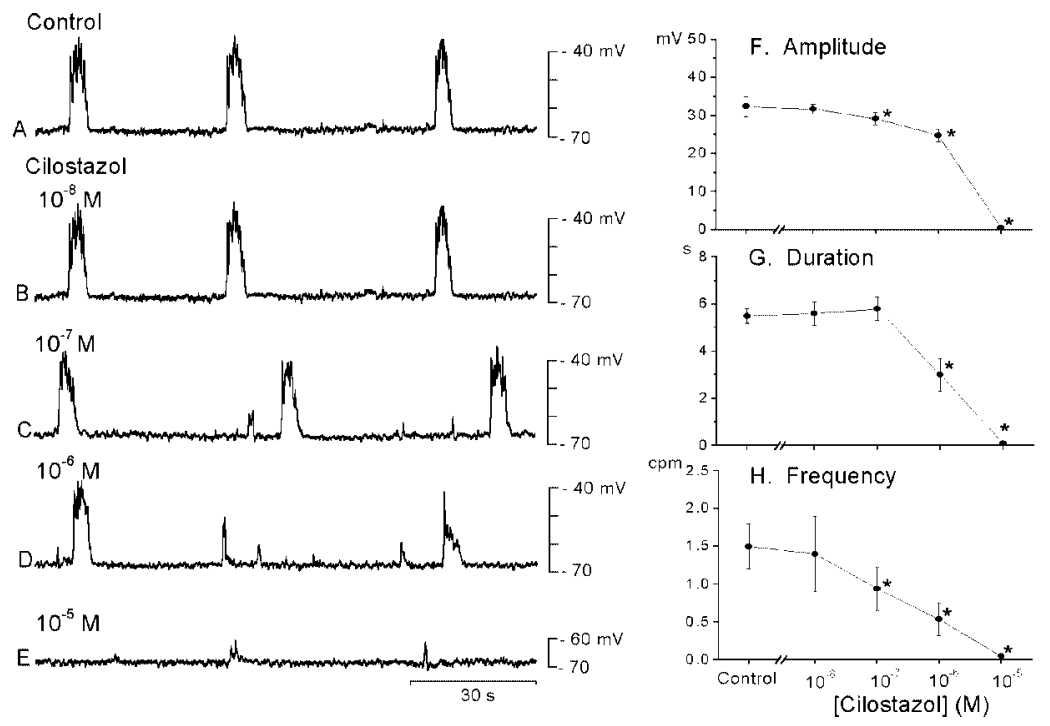

Fig. 2. Effects of cilostazol on slow potentials recorded from an isolated circular muscle preparation. Membrane potentials were recorded in the absence (A) and presence of cilostazol (B, 10-8 M; C, $10^{-7} \mathrm{M}$; D, $10^{-6} \mathrm{M}$; E, $10^{-5} \mathrm{M}$ ). Records A-E were obtained from a single cell. The graphs at the right-hand side shows the concentrationresponse relationship of the effects of cilostazol on amplitude in $\mathrm{mV}(\mathrm{F})$, duration in $\mathrm{s}$ $(\mathrm{G})$ and frequency in $\mathrm{cpm}(\mathrm{H})$ of slow potentials recorded from circular smooth muscle cells. Mean \pm S.D. $(n=6-12)$. *: signifies statistical significant difference from control as determined by the Student's $t$-test $(\mathrm{P}<0.05)$.

cilostazol, $1.22 \pm 0.56 \mathrm{cpm}, n=9$ ), with no significant alteration of the amplitude (control, $28.7 \pm$ $1.5 \mathrm{mV}$; in cilostazol, $27.1 \pm 1.9 \mathrm{mV}, n=9$ ) (Fig. 3A). In a given pair of cells, evoked slow potentials generated in response to depolarization produced by current pulses applied via another electrode were recorded in the absence and presence of $10^{-7} \mathrm{M}$ cilostazol (Fig. 3, B and $\mathrm{C}$, respectively). The electrical stimuli were applied to smooth muscle cells, and the amplitude of the evoked response was plotted as a function of time after the cessation of spontaneous slow potentials. The results showed an identical relationship between the responses in the absence and presence of cilostazol (Fig. 3D). This suggests that the inhibition of slow potentials by cilostazol was not due to an enhanced refractory period for the generation of slow potentials.

The effects of cilostazol on the input resistance of the smooth muscle membrane were examined in segments containing circular muscle bundles using paired microelectrodes. Inward current pulses produced electrotonic potentials in an intensity-dependent way, while outward current pulses produced electrotonic potentials which evoked slow potentials at higher intensities, either in the absence (Fig. 4A) or presence of $10^{-5} \mathrm{M}$ cilostazol (Fig. 4B). The current-voltage relationship showed that it was not altered by cilostazol (Fig. 4C). The input resistance calculated from the current-voltage relationship $(1.91 \pm 0.11 \mathrm{M} \Omega, n=5)$ was not changed in the presence of cilostazol $(1.85 \pm 0.04 \mathrm{M} \Omega, n=5, \mathrm{P}>0.05)$. These results indicated that the inhibition of spontaneous activity by cilostazol was not causally related to the change in input resistance of the smooth muscle membrane. 

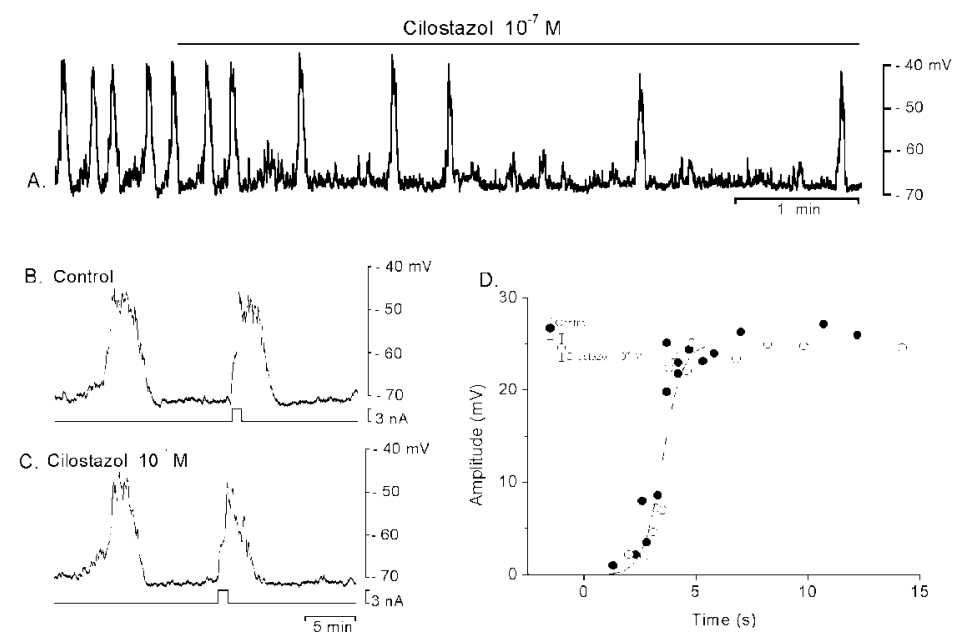

Fig. 3. Effects of cilostazol on the refractory period for excitation of circular smooth muscle cells. A: Electrical responses were recorded from an isolated circular muscle preparation isolated from the guinea-pig stomach antrum, in the presence of $10^{-7} \mathrm{M}$ cilostazol for the duration of the bar shown above the record. B-D: Electrical responses were recorded simultaneously from a given pair of smooth muscle cells in a small segment of circular muscle tissue (about $100 \mu \mathrm{m} \times 250 \mu \mathrm{m}$ ), and muscle was stimulated by a depolarizing current pulse (duration, $1 \mathrm{~s}$; intensity, $3 \mathrm{nA}$ ) at about $7 \mathrm{~s}$ after the cessation of spontaneously generated slow potentials, in the absence (B, Control) and presence of $10^{-7} \mathrm{M}$ cilostazol (C). D shows the relationship between the time for stimulation with depolarizing pulses after cessation of slow potentials and amplitude of the evoked responses, in the absence (filled circles) and presence of $10^{-7}$ M cilostazol (open circles). Amplitude of slow potentials generated spontaneously (mean \pm S.D.) are shown at the left-hand side of the graphs.

\section{Effects of cilostazol on tissue cAMP content}

As cilostazol elevates the tissue cAMP content in vascular smooth muscle cells due to inhibition of phosphodiesterases (Kawamura et al., 1985; Kimura et al., 1985; Kohda et al., 1999), attempts were made to measure the content of cAMP in antrum smooth muscle tissue. For comparison, the effects of $10^{-3} \mathrm{M}$ caffeine and $10^{-7} \mathrm{M}$ forskolin, which are both known to elevate tissue cAMP levels (Arnaud, 1987), were also examined. The results of a series of experiments are summarized in Fig. 5. The level of cAMP was significantly increased by an average of about $30 \%$ in preparations stimulated with $10^{-5} \mathrm{M}$ cilostazol, but not at concentrations of $10^{-7}$ or $10^{-6} \mathrm{M}$, in comparison with those stimulated with DMSO, a solvent of cilostazol (Fig. $5 \mathrm{~A})$. The tissue levels of cAMP were also increased significantly by forskolin, in comparison with those of DMSO alone (Fig. 5B). Tissues stimulated with caffeine produced elevated cAMP contents, but not significantly $(0.05<\mathrm{P}<0.1)$ compared to those incubated in Krebs solution alone (Fig. 5C).

Comparison of the effects of cilostazol, caffeine and forskolin on electrical activity of gastric smooth muscle

The effects of $10^{-5} \mathrm{M}$ cilostazol, $10^{-3} \mathrm{M}$ caffeine and $10^{-7} \mathrm{M}$ forskolin on slow waves were 

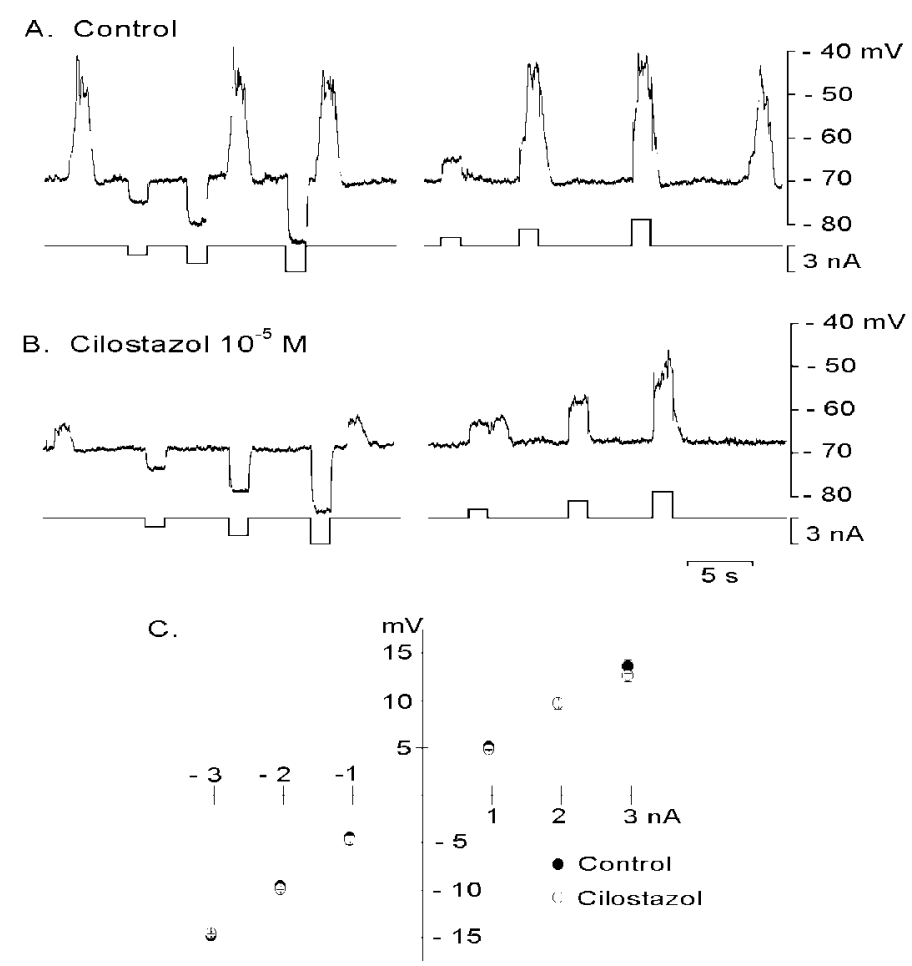

Fig. 4. Effects of cilostazol on the input resistance of circular smooth muscle cells. In a small segment of circular muscle tissue (about $100 \mu \mathrm{m} \times 250 \mu \mathrm{m}$ ), electrical responses were recorded simultaneously from a given pair of cells, and depolarizing and hyperpolarizing current pulses (duration, $1 \mathrm{~s}$; intensity, 1-5 nA) were applied to one electrode and electrotonic potentials generated by the pulses were recorded from the second electrode, in the absence (A) and presence of $10^{-7} \mathrm{M}$ cilostazol (B). C: The relationship between intensity of inward current pulses and amplitude of electrotonic potentials, recorded in the absence (Control, filled circles) and presence of $10^{-7} \mathrm{M}$ cilostazol (open circles). Mean \pm S.D. $(n=5-6)$.

compared, and the results summarized in Fig. 6. The typical examples shown in Fig. 6 (A-D) indicate that the amplitude and frequency of slow waves was significantly reduced by caffeine but not by cilostazol and forskolin. The summarized data obtained from 5-12 preparations indicates that the amplitude of slow waves was significantly reduced by cilostazol, caffeine and forskolin, in the order of caffeine $>$ forskolin $>$ cilostazol (Fig. 6 E). The duration of slow waves was significantly reduced by caffeine but not by cilostazol and forskolin (Fig. 6 F). The frequency of slow waves was significantly inhibited by caffeine and forskolin, but not by cilostazol (Fig. 6G).

As shown in Fig. 7 (A-D), the comparison of the effects of cilostazol, caffeine and forskolin on typical examples of follower potentials indicated that significant change appeared only on the reduction in duration. Summarized data obtained from 4-8 preparations indicates that the amplitude of follower potentials was not significantly modulated by cilostazol, caffeine or forskolin (Fig. 7E). Significant reduction of the duration of follower potentials was produced by 
A

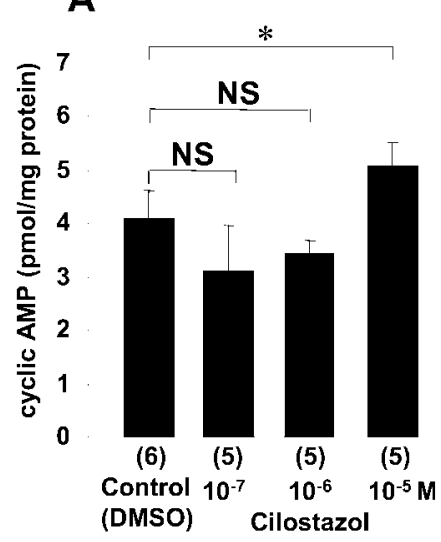

B

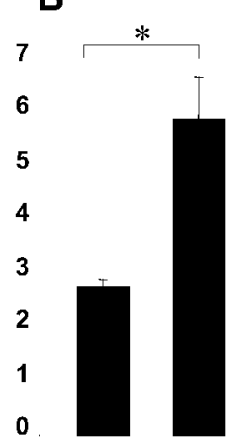

(6)

(5)

Control $10^{-7} \mathrm{M}$

(DMSO) Forskolin

C

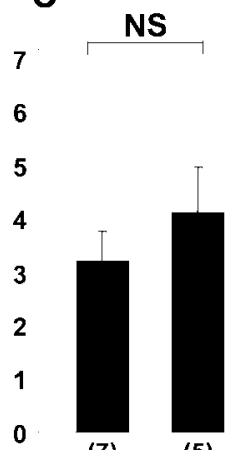

(7) (5)

Control $10^{-3} \mathrm{M}$

(Krebs) Caffeine

Fig. 5. Effects of cilostazol, caffeine and forskolin on cyclic AMP content. Tissue levels of cyclic AMP were measured in the presence of cilostazol (A), forskolin (B) and caffeine $(\mathrm{C})$, in smooth muscle of the stomach antrum. Control solution contained $0.01 \mu \mathrm{l} / \mathrm{ml}$ either DMSO or water in Krebs solution as the control for cilostazol and forskolin or for caffeine, respectively. Mean \pm S.E.M. Number of preparations is given in the parentheses shown at the foot of each column. *: $\mathrm{P}<0.05$ by Williams' test for cilostzol and Student's $t$-test for forskolin and caffeine. NS: not significant $(\mathrm{P}>0.05)$.

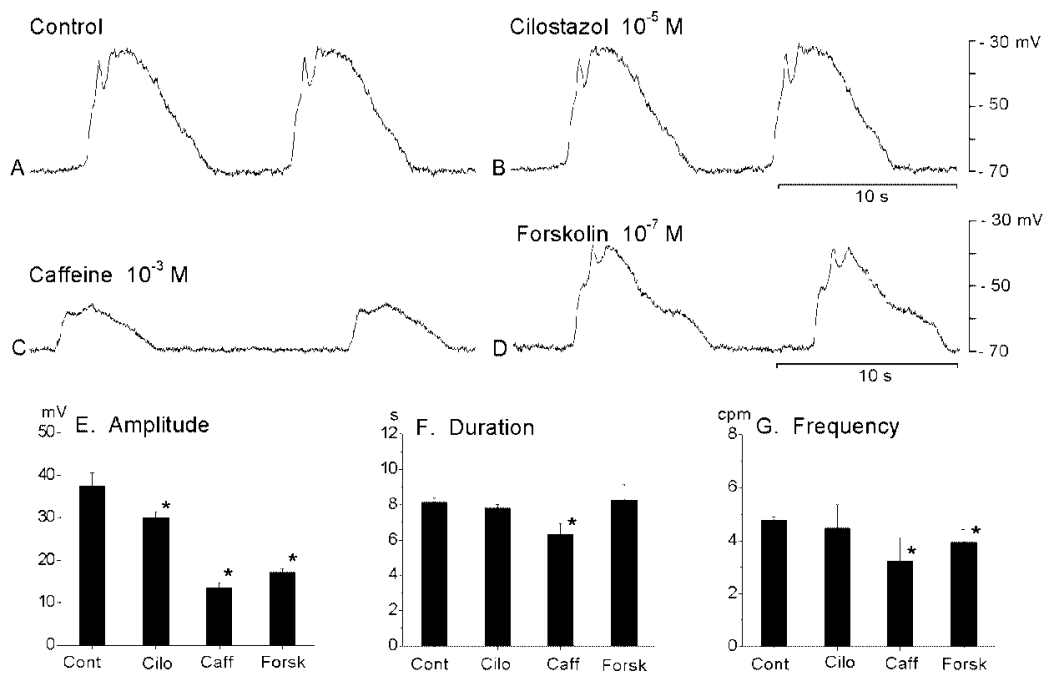

Fig. 6. The comparison of the effects of cilostazol, caffeine and forskolin on slow waves. Typical examples of slow waves recorded from circular smooth muscle cells of intact tissue, in control conditions (A) or in the presence of either $10^{-5} \mathrm{M}$ cilostazol (B), $10^{-3}$ $\mathrm{M}$ caffeine (C) or $10^{-7} \mathrm{M}$ forskolin (D). Amplitude in $\mathrm{mV}(\mathrm{E})$, duration in $\mathrm{s}(\mathrm{F})$ and frequency of slow waves in cpm $(\mathrm{G})$ recorded in the control (Cont) and in the presence of either cilostazol (Cilo), caffeine (Caff) or forskolin (Forsk) were shown by the mean \pm S.D. $(n=5-12)$. *: indicates statistical significance from control as determined by Student's $t$-test. 


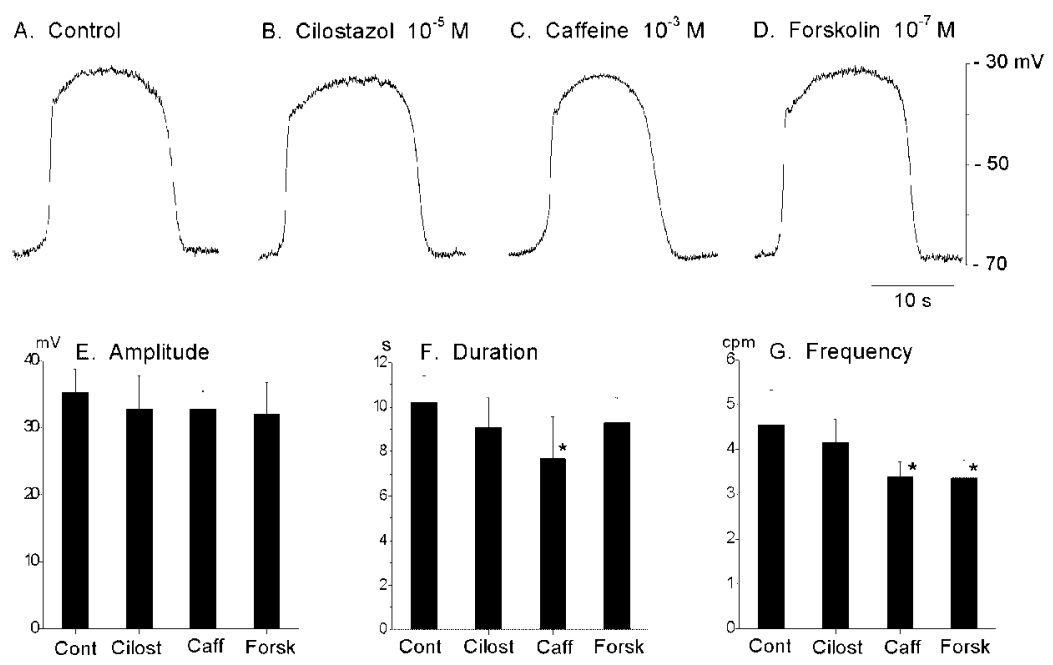

Fig. 7. Effects of cilostazol, caffeine and forskolin on follower potentials. Typical examples of follower potentials were recorded in the control (A) or in the presence of either $10^{-5}$ $\mathrm{M}$ cilostazol (B), $10^{-3} \mathrm{M}$ caffeine (C) or $10^{-7} \mathrm{M}$ forskolin (D). Mean values ( \pm S.D.) of the amplitude in $\mathrm{mV}(\mathrm{E})$, duration in $\mathrm{s}(\mathrm{F})$ and frequency of follower potentials in $\mathrm{mV}$ (G) recorded from 4-8 tissues are summarized. *: indicates statistical significance from control as determined by Student's $t$-test.

caffeine (Fig. 7F). The frequency of follower potentials was significantly reduced by caffeine and forskolin, with the potency in the order of caffeine $>$ forskolin (Fig. 7G). Thus, the effects of cilostazol on follower potentials clearly differed from those of caffeine and forskolin.

\section{Discussion}

The present experiments have shown that in the smooth muscle cells of the guinea-pig stomach antrum, cilostazol reduces the amplitude of slow waves with no marked alteration to the pacemaker potentials and follower potentials. Slow waves are formed of $1^{\text {st }}$ and $2^{\text {nd }}$ components (Tomita, 1981), each of which is formed respectively by the propagated electrotonic potential of pacemaker potentials and regenerative slow potentials that are generated in circular muscle cells (Suzuki and Hirst, 1999; Suzuki, 2000; Hirst and Ward, 2003). The inhibition by cilostazol of slow waves appeared mainly on the regenerative $2^{\text {nd }}$ component, suggesting that the activity of the circular smooth muscle was mainly inhibited. This was supported by the difference in the effects of cilostazol between slow potentials and pacemaker or follower potentials, in that cilostazol inhibited slow potentials at concentrations of $10^{-7} \mathrm{M}$, while it inhibited pacemaker potentials or follower potentials only weakly at $10^{-5} \mathrm{M}$. Thus, cilostazol selectively inhibits slow potentials generated in the circular smooth muscle.

Slow potentials generated in circular muscle bundles are formed by the summation of unitary potentials which may be propagated from intramuscular interstitial cells of Cajal (ICCIM) in an electrotonic manner (Edwards et al., 1999; Hirst and Ward, 2003). In the present experiments, cilostazol did not markedly alter the resting membrane potential of smooth muscle 
cells, suggesting that the ionic conductance of the membrane was not markedly changed. This was confirmed by the absence of any significant change in the input resistance of the membrane during the inhibition of spontaneous activity with cilostazol. Ca-activated Cl-conductances may be taking the central role in the formation of pacemaker potentials (Tokutomi et al., 1995; Kito et al., 2002a) or slow potentials (Hirst et al., 2002), and thus the absence of significant inhibition of pacemaker potentials by cilostazol suggests that this chemical does not have a significant ability to inhibit Ca-activated Cl-channels. The refractory period for the generation of slow potentials has a causal relation to the activity of protein kinase C (PKC) (Kito et al., 2002b), and inhibition of PKC decreases while activation of PKC with phorbol esters increases the frequency of spontaneously generating slow potentials (Nakamura and Suzuki, 2003; Lee et al., 2004). As cilostazol did not alter the refractory period, no alteration to the activity of PKC was produced in pacemaker cells distributed within the circular muscle bundles (possibly ICC-IM).

Cilostazol has clinical importance due to inhibition of platelate aggregation and peripheral vasodilatation via elevation of cAMP (Kawamura et al., 1985; Kimura et al., 1985; Kohda et al., 1999). The present experiments revealed that the elevation of cAMP by $10^{-5} \mathrm{M}$ cilostazol was significantly smaller in gastric muscle (equal to about $5 \mathrm{pmol} / \mathrm{mg}$ protein) than in vascular smooth muscle cells (equal to about $200 \mathrm{pmol} / \mathrm{mg}$ protein; read from Fig. 6 in Hayashi et al., 2000), suggesting that cilostazol may have high selectivity in vascular tissues. These results allow speculation that the elevation by cilostazol of the level of cAMP in gastric muscles is largely produced in vascular tissues distributed in the gastric wall.

The concentration of cilostazol required to elevate the level of cAMP in gastric muscle tissue was $10^{-5} \mathrm{M}$, while the inhibition of electrical activity in the circular muscle appeared at $>10^{-7} \mathrm{M}$ cilostazol. These differences in effective concentration of cilostazol could suggest that the inhibition of electrical activity was not causally related to the elevation of cAMP. Many types of phosphodiesterase inhibitors equally inhibit spontaneous electrical and mechanical activities of gastric muscles (Tsugeno et al., 1995). However, absence of any causal relation to the inhibitory actions of caffeine and the tissue contents of cAMP are reported in the guinea-pig small intestine (Prestwich and Bolton, 1995). This seems to be also the case for the actions of cilostazol on gastric smooth muscle.

One of the characteristics of the inhibitory actions of cilostazol on gastric muscle was that the inhibition of slow waves was evident on the amplitude, and only weakly on the frequency and duration. The inhibition of the amplitude of slow waves may be mainly due to the reduced amplitude of the $2^{\text {nd }}$ component of slow waves. The frequency and duration of slow waves are determined passively, in response to changes in pacemaker potential. This means that the inhibitory actions of cilostazol appear to occur mainly on circular muscle cells, and this may differ from the actions of caffeine and forskolin, as both of the latter chemicals inhibit the frequency of slow waves, in addition to the amplitude or duration of slow waves. Thus, the pacemaker mechanism is inhibited by caffeine and forskolin but not by cilostazol. The amplitude of slow waves was inhibited by caffeine and forskolin, with no significant inhibition of follower potentials, suggesting that both of these chemicals inhibited the activity of circular muscle cells more selectively than that of the pacemaker cells. This was similar to the action of cilostazol, but the inhibition by caffeine and forskolin are much stronger than cilostazol. 
Follower potentials are formed by passively propagated pacemaker potentials through gap junctions, and the absence of significant inhibition by cilostazol of these potentials suggests that the gap junctional communications are not altered by cilostazol. This also seemed to be the case for caffeine and forskolin, since there was a parallel change in the duration and frequency of slow waves and follower potentials. Caffeine inhibited the frequency and duration in both slow waves and follower potentials.

It is concluded that in the antrum smooth muscle of the stomach, the inhibitory actions of cilostazol appear mainly on the circular muscle cells and the ICC-IM, with no causal relation to the tissue content of cAMP. The effects of cilostazol on pacemaker potentials are very weak, and as a consequence the inhibition appears mainly on the amplitude but not the frequency of slow waves. Caffeine and forskolin have much stronger inhibitory effects on both smooth muscle cells and pacemaker cells than cilostazol does, with an associated increase in the tissue level of cAMP for forskolin and no significant change for caffeine. These effects produce features characteristic to these chemicals, i.e., the amplitude and frequency of slow waves are inhibited. The present experiments could not find any causal relation between the inhibitory actions of cilostazol, caffeine and forskolin on gastric smooth muscle cells and the elevation of the tissue contents of cAMP.

\section{References}

Abdel-Latif, A.A. (1996). Cross talk between cyclic AMP and the polyphosphoinositide signaling cascade in iris sphincter and other nonvascular smooth muscle. Proc. Soc. Exp. Biol. Med. 211: 163-177.

Arnoud, M.J. (1987). The pharmacology of caffeine. Prog. Drug Res. 31, 273-313.

Berridge, M.J. (1993). Inositol trisphosphate and calcium signaling. Nature 361: 315-325.

Dawson, D.L., Cutler, B.S., Meissner, M.H. and Strandness, D.E. Jr. (1998). Cilostazol has beneficial effects in treatment of intermittent claudication: results from a multicenter, randomized, prospective, double-blind trial. Circulation 98: 678-686.

Dickens, E.J., Hirst, G.D.S. and Tomita, T. (1999). Identification of rhythmically active cells in guinea-pig stomach. J. Physiol. (Lond.) 514: 515-531.

Edwards, F.R., Hirst, G.D.S. and Suzuki, H. (1999). Unitary nature of regenerative potentials recorded from circular smooth muscle of guinea-pig antrum. J. Physiol. (Lond.) 519: 235-250.

Fukuta, H., Kito, Y. and Suzuki, H. (2002). Spontaneous electrical activity and associated changes in calcium concentration in guinea-pig gastric smooth muscle. J. Physiol. (Lond.) 540: 249-260.

Hayashi, S., Morishita, R., Matsushita, H., Nakagami, H., Taniyama, Y., Nakamura, T., Aoki, M., Yamamoto, K., Higaki, J. and Ogihara, T. (2000). Cyclic AMP inhibited proliferation of human aortic vascular smooth muscle cells, accompanied by induction of p53 and p21. Hypertension 35 : 237-243.

Hirst, G.D.S., Bramich, N.J., Teramoto, N., Suzuki, H. and Edwards, F.R. (2002). Regenerative component of slow waves involves a delayed increase in $\left[\mathrm{Ca}^{2+}\right]_{\mathrm{i}}$ and $\mathrm{Cl}^{-}$channels. J. Physiol. (Lond.) 540: 907-919.

Hirst, G.D.S. and Ward, S.M. (2003). Interstitial cells: involvement in rhythmicity and neural control of gut smooth muscle. J. Physiol. (Lond.) 550: 337-346.

Huizinga, J.D., Farraway, L. and Den Hertog, A. (1991). Effect of voltage and cyclic AMP on frequency of slow-wave-type action potentials in canine colon smooth muscle. J. Physiol. (Lond.) 442: 31-45.

Huizinga, J.D., Thuneberg, L., Vanderwinden, J.M. and Rumessen, J. (1997). Interstitial cells of Cajal as targets for pharmacological intervention in gastrointestinal motor disorders. Trends Pharmacol. 
Sci. 8: 393-403.

Kawamura, K., Watanabe, K. and Kimura, Y. (1985). Effect of cilostazol, a new antithrombotic drug, on cerebral circulation. Arzeimittelforsch. 35: 1149-1154.

Kimura, Y., Tani, T., Kanbe, T. and Watanabe, K. (1985). Effect of cilostazol on platelet aggregation and experimental thrombosis. Arzneimittelforsch. 35: 1144-1149.

Kito, Y., Fukuta, H. and Suzuki, H. (2002a). Components of pacemaker ptentials recorded from the guinea-pig stomach antrum. Pflügers Arch. 445: 202-217.

Kito, Y., Fukuta, H., Yamamoto, T. and Suzuki, H. (2002b). Excitation of smooth muscle isolated from circular muscle of the guinea-pig gastric antrum in response to depolarization. J. Physiol. (Lond.) 543: 155-167.

Kito, Y. and Suzuki, H. (2003a). Pacemaker frequency is increased by sodium nitroprusside in the guinea-pig gastric antrum. J. Physiol. (Lond.) 546: 191-205.

Kito, Y. and Suzuki, H. (2003b). Electrophysiological properties of gastric pacemaker potentials. $J$. Smooth Muscle Res. 39: 163-173.

Kito, Y. and Suzuki, H. (2003c). Properties of pacemaker potentials recorded from myenteric interstitial cells of Cajal distributed in the mouse small intestine. J. Physiol. (Lond.) 553: 803-818.

Kobayashi, S., Yamaguchi, S., Katsube, T., Kitani, M., Okada, K. and Tsunematsu, T. (1985). Long-term effect of cilostazol on cerebral blood flow in chronic cerebral infarction. Arzneimittelforsch. 35 : 1193-1197.

Koh, S.D., Kim, T.W., Jun, J.Y., Glasgow, N.J., Ward, S.M. and Sanders, K.M. (2000). Regulation of pacemaker currents in interstitial cells of Cajal from murine small intestine by cyclic nucleotides. J. Physiol. (Lond.) 527: 149-162.

Kohda, N., Tani, T., Nakayama, S., Adachi, T., Marukawa, K., Ito, R., Ishida, K., Matsumoto, Y. and Kimura, Y. (1999). Effect of cilostazol, a phosphodiesterase III inhibitor, on experimental thrombosis in the porcine carotid artery. Thromb. Res. 96: 261-268.

Komuro, T., Tokui, K. and Zhou, D.S. (1996). Identification of the interstitial cells of Cajal. Histol. Histopathol. 11: 769-786.

Kuriyama, H., Kitamura, K., Itoh, T. and Inoue, R. (1998). Physiological features of visceral smooth muscle cells, with special reference to receptors and ion channels. Physiol. Rev. 78: 811-920.

Lee, K.P., Nakamura, E., So, I., Kim, K.W. and Suzuki, H. (2004). Role of protein kinase C in the excitatory actions of cholinergic nerve stimulation on spontaneous activity of circular smooth muscle isolated from the guinea pig stomach antrum. Pflugers Arch. (in press).

Maes, K., Missiaen, L., Parys, J.B., Sienaert, I., Bultynck, G., Zizi, M., De Smet, P., Casteels, R. and De Smedt, H. (1999). Adenine-nucleotide biding sites on the inositol 1,4,5-trisphosphate receptor bind caffeine, but not adenophostin A or cyclic ADP-ribose. Cell Calcium 25: 143-152.

Nakamura, E. and Suzuki, H. (2003). Effects of protein kinase C inhibitors on the frequency of spontaneous activity in circular smooth muscle isolated from the guinea-pig stomach. J. Smooth Muscle Res. 7: J30 (Japanese abstract).

Nose, K., Suzuki, H. and Kannan, H. (2000). Voltage-dependency of the frequency of slow waves in antrum smooth muscle of the guinea-pig stomach. Jpn. J. Physiol. 50: 625-633.

Ozaki, H., Blondfield, D.P., Hori, M., Sanders, K.M. and Publicover, N.G. (1992). Cyclic AMP-mediated regulation of excitation-contraction coupling in canine gastric smooth muscle. J. Physiol. (Lond.) 447: 351-372.

Parker, I. and Ivorra, I. (1991). Caffeine inhibits inositol trisphosphate-mediated liberation of intracellular calcium in Xenopus oocytes. J. Physiol. (Lond.) 433: 229-240.

Prestwich, S.A. and Bolton, T.B. (1995). Inhibition of muscarinic receptor-induced inositol phospholipid hydrolysis by caffeine, $\beta$-adrenoceptors and protein kinase $\mathrm{C}$ in intestinal smooth muscle. $\mathrm{Br}$. J. Pharmacol. 114: 602-611.

Sanders, K.M. (1996). A case for interstitial cells of Cajal as pacemakers and mediators of 
neurotransmission in the gastrointestinal tract. Gastroenterol. 111: 492-515.

Sanders, K.M., Ördög, T., Koh, S.D., Torihashi, S. and Ward S.M. (1999). Development and plasticity of interstitial cells of Cajal. Neurogastroenterol. Motil. 11:311-338.

Somlyo, A.P. and Somlyo, A.V. (1994). Signal transduction and regulation in smooth muscle. Nature 372: 231-236.

Suzuki, H. and Hirst, G.D.S. (1999). Regenerative potentials evoked in circular smooth muscle of the antral region of guinea-pig stomach. J. Physiol. (Lond.) 517: 563-573.

Suzuki, H. (2000). Cellular mechanisms of myogenic activity in gastric smooth muscle. Jpn. J. Physiol. 50: 289-301.

Takaki, M. (2003). Gut pacemaker cells: the interstitial cells of Cajal (ICC). J. Smooth Muscle Res. 39: 137-161.

Tanaka, K., Gotoh, F., Fukuuchi, Y., Amano, T., Uematsu, D., Kawamura, J., Yamawaki, T., Itoh, N., Obara, K. and Muramatsu, K. (1989). Effects of selective inhibitor of cyclic AMP phosphodiesterase on the pial microcirculation in feline cerebral ischemia. Stroke 20: 668-673.

Tertyshnikova, S. and Fein, A. (1998). Inhibition of inositol 1,4,5-trisphosphate-induced $\mathrm{Ca}^{2+}$ release by cAMP-dependent protein kinase in a living cell. Proc. Natl. Acad. Sci. U S A. 95: 1613-1617.

Toescu, E.C., O’Neill, S.C., Petersen, O.H. and Eisner, D.A. (1992). Caffeine inhibits the agonist-evoked cytosolic $\mathrm{Ca}^{2+}$ signal in mouse pancreatic acinar cells by blocking inositol trisphosphate production. J. Biol. Chem. 267: 23467-23470.

Tokutomi, N., Maeda, H., Tokutomi, Y., Sato, D., Sugita, M., Nishikawa, S., Nishikawa, S., Nakao, J., Imamura, T. and Nishi, K. (1995). Rhythmic $\mathrm{Cl}^{-}$current and physiological roles of the intestinal c-kit-positive cells. Pflügers Arch. 431: 169-177.

Tomita, T. (1981). Electrical activity (spikes and slow wave) in gastrointestinal smooth muscles. In: Smooth Muscle, ed. by Bülbring, E., Brading, A.F., Jones, A.W., and Tomita, T., Edward Arnold, London, pp. 127-156.

Tsugeno, M., Huang, S.M., Pang, Y.W., Chowdhury, J.U. and Tomita, T. (1995). Effects of phosphodiesterase inhibitors on spontaneous electrical activity (slow waves) in guinea-pig gastric muscle. J. Physiol. (Lond.) 485: 493-502.

Yasuda, K., Sakuma, M. and Tanabe, T. (1985). Hemodynamic effect of cilostazol on increasing peripheral blood flow in arteriosclerosis obliterans. Arzneimittelforsch. 35: 1198-1200.

(Received May 31, 2004; Accepted June 18, 2004) 